\title{
A Research on Kevlar and Hybrid Kevlar Composites; A Report
}

\author{
Mohammed Hisham, Mohammed Fahaduddin, Mohammed Azhar Khan, Ashok B C, Prashant \\ Kumar Shrivastava
}

\begin{abstract}
This study focused on the Kevlar fiber composite, the demand of Kevlar composites increasing day-byday because it's light weight and good mechanical properties. There are different types of fiber composites are available like Carbon, Basalt, Glass, Jute, Kenaf, Flax, Hemp and Kevlar etc. Out of these available material Kevlar is one of the most favorable composite material. Properties of Kevlar include high rigidity modulus, toughness, thermal stability and most importantly strength. Moreover, the properties of Kevlar composite can be increased by applying the different hybridization and treatment process. The aim of this study, to explore the different types of hybridization and treatments that can be applied for improving the mechanical properties of Kevlar composite.
\end{abstract}

Keywords - Kevlar, hybridization, treatments, composite

\section{INTRODUCTION}

There are many researchers continues work on the engineering material that having good mechanical properties, light in weight and cost effective. The advanced engineering material like Inconel and titanium and its alloys are highly demanded material for different industries like, automobile, flooding, navy, defense, aerospace and space industries. The engineering materials generally divided in to three categories like metals, non-metals and composites. These material having their own merits and demerits according to the uses. The selection of the engineering material differs according to requirement of the industries [1]-[4]. The classification of engineering material shown in Fig. 1.

These are the available engineering material in the market. Recently, scenario has been changed the demand of composite and alloy material increased because of its great mechanical properties. There are three types of composite material according to the composite material like metal composite, powder composites and fiber composites. Nowadays, in different industries demand of fiber composites increasing day-by-day because these materials

Revised Manuscript Received on July 10, 2019

Mohammed Hisham, Department of Mechanical Engineering, Vidyavardhaka College of Engineering Mysuru, Karnataka. India. (E-mail:

Mohammed Fahaduddin, Department of Mechanical Engineering, Vidyavardhaka College of Engineering Mysuru, Karnataka. India. (E-mail: mohammedfahadcr8@gmail.com)

Mohammed Azhar Khan, Department of Mechanical Engineering, Vidyavardhaka College of Engineering Mysuru, Karnataka. India. (E-mail: mohammedazharkhan335@gmail.com) College of Engineering Mysuru, Karnataka. India. (E-mail: bcashok@vvce.ac.in)

Prashant Kumar Shrivastava, Department of Mechanical Engineering, Vidyavardhaka College of Engineering Mysuru, Karnataka India. (E-mail: er.prashant1986@gmail.com) mdhisham1998@gmail.com)

Ashok B C, Department of Mechanical Engineering, Vidyavardhaka

are light in weight and having good mechanical properties. There are different types of fiber composites are available like carbon, basalt, glass, jute, kenaf, flax, hemp and Kevlar etc. Out of these available material kevlar is one of the most favorable composite material. Properties of Kevlar include high rigidity modulus, toughness, thermal stability and most importantly strength.

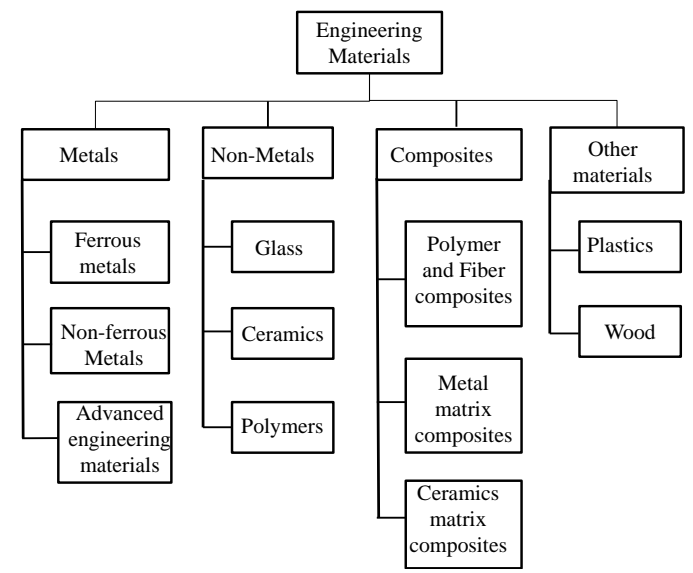

Figure 1. Classification of engineering material

Kevlar gets its high strength from numerous inter-chain bonds. When it is spun, Kevlar is said to have a relative density of 1.44 and strength of 3260MPa. The inter-chain bonds are formed between $\mathrm{NH}$ centers and carbonyl groups and these chain lengths have an effect on other synthetic polymers' properties like Dyneema. Even in temperatures such as $-196{ }^{\circ} \mathrm{C}$ (Cryogenic temperatures) Kevlar retains its resilience and strength and to some degree a bit stronger at lower temperatures. Kevlar has a wide range of application from race cars to bullet proof armours etc.

Racing cars are prone to accidents. Kevlar is used to reinforce its fuel tanks due to its tensile strength. This makes the fuel tank difficult to be punctured in case of an impact or an accident resulting in a low risk of fire during a crash. And since the property of Kevlar is being so light, it is an ideal material. Traditionally Steel was the material of choice for body armour. But it was bulky and heavy. Kevlar on the other hand is light weight and 18 times stronger than steel. Due to Kevlar's high tensile strength it is easy for it to absorb high impact energy. Hence it is the best suited material for protection against knife hits and bullets. Kevlar cables are a good replacement for steel cables as they are much stronger, flexible and light weight. 


\section{LITERATURE SURVEY}

The purpose of this research is to establish stab proof material made up of shear thickening fluid (STF) and Kevlar fiber. In this research, silica / ethylene glycol suspension was prepared for the use as STF and it was evaluated by rheometer. From the results, it was seen that STF exhibited a reverse liquid- solid transition at a particular shear. Kevlar was treated as STF by 1 dip 1 nip method and mechanical and stab resistant properties were analyzed. After viewing both the results, STF impregnation comprehensively upgraded the stab resistance of Kevlar against the spike threats and the safety aspect of Kevlar was also increased comprehensively. The stab resistant coating was performed by considering one STF, fumed silica / ethylene glycol suspension of Kevlar fabric to enhance the performance of the material. From this research, extensive upgrades in puncture defiance were seen especially in excessive speed loading condition. It was seen that the addition did not change or deteriorate the flexibility of STF. From the results, we incurred that fumed silica/ Kevlar composite fabric would be a fine material for body armored applications [5].

Moreover, multiple materials that are combined to produce an individual component. The two materials that were investigated were Kevlar poly (p-phenylene terephthalamide) and santoprene. Here in this investigation, the Kevlar fiber was used in two ways, the first type was, it was used without altering it and the second type was used after altering it. The Kevlar which was used without modifying strengthened the santoprene to quote an extent and it upgraded few properties of the composite, namely low strain modulus and tensile strength but it also had a drawback, lengthening at break reduced heavily. In order to overcome this, the Kevlar was modified and hence its surface was hydrolyzed maleic anhydride-graftedpolypropylene (MA-g-PP). There were clear advantages of using the modified Kevlar over the stock one. The properties enhanced and the drawbacks were reduced to nil. This combination showed improved stress distribution due to better surface bonding between the fiber and matrix [6].

Thereafter, Fluorinated and Oxy fluorinated Short Kevlar Fiber-Reinforced Ethylene Propylene Polymer This paper examines raw Kevlar and surface treated Kevlar. Examination on its thermal properties showed an increase in thermal stability and storage modulus due to gradual reinforcement of fibers. It is also noted that it continues to increase in the case of ox fluorinated and fluorinated Kevlar fiber-reinforced EP. Fluorinated and oxy fluorinated Kevlar fiber's tensile strength increased substantially. This concludes that fluorination and oxy fluorination had an impact on the surface morphology giving better adhesion of fibers and the matrix[7]

Moreover, comparison of properties like crystalline, thermal, mechanical of syndiotatic polystyrene composites with surface modified Kevlar fiber. In comparison to oxyfluorinated Kevlar reinforced fiber, fluorinated Kevlar fiber reaches higher crystallization temperature. There is a significant increase in thermal conductivity in case of modified Kevlar fiber reinforcement. It also shifts to a higher value shown by differential scanning calorimeter and dynamic mechanical analysis. A strong adhesion between s-
PS matrix and oxy-fluorinated Kevlar fiber was found and it seems to be better than other composites shown by atomic free microscopy. From this research it was found that it boosts up the thermal stability and storage modulus of the composite [7]

However, the properties of Kevlar polypropylene based on composite material under high strain rate loading using Split Hopkinson Pressure bar (SHPB). Flat laminated Kevlar composite of 16, 24 and 30 layer where comparison molded and laser machined to obtain cylindrical specimen of desired shape based on SHPB experiment. To report compressive material behavior as expansion of growing strain rate, the stress strain plots were obtain and analyzed. The studies suggest that for better performance of composite laminates apply thin laminates. The $90 \%$ of thickness composite depend on the peak stress, strain and toughness. On the basis of high strain rate testing of 16, 24, 30 layered Kevlar polypropylene composite SHPB testing was done and the following conclusion were made. At low strain rates the growth pattern of stress and strain are linear and further continues the same nature even at high strain rates. As per research the thin specimen are more useful than the thick specimen [8]

Moreover, laminated hybrid and unidirectional composites, and looks into the effects of carbon to Kevlar ratio on mechanical properties. Bismaleimide (BMI) resin is reinforced from hybridizing 3-D braided carbons and Kevlar fibers. This braided carbon/Kevlar showed higher flexure strength, which was expected. Linearity was noticed, but only on attaining peak values of loading. It attained maximum flexure strength when the carbon to Kevlar ratio was 3:2. Addition of ductile fiber improved its impact properties significantly of all carbon composites. As the volume of the relative Kevlar fiber increased, residual flexure and energy absorption also increased which were tested with the impact samples[9].

Thereafter, finding an alternative for soft Kevlar that is more durable and safe. This was done by introducing shear stiffening gel (STG) into shear thickening fluid (STF) impregnated Kevlar woven fabric. It improved its impact resistance do to its good shear stiffening characteristics. A number of experiments were conducted such as knife cutting test, yarn pull out test, rod penetration test etc. to find out its improvement. STG which was devised into the gap of the filament increased friction. STG had increased the modulus of the yarn and the multi layered fabric had a reduction in its compressibility. Impact test such as the drop hammer test showed that STG increased the impact resistance force and also its deformation displacement. This leads to a better knife jab resistance compared to Kevlar. The Kevlar/STG/STF also had lower weight compared to Kevlar. In conclusion the enhanced Kevlar fabric is more light weight and gives very good impact resistance hence ideal for soft body armour [10].

Moreover, the effects of low velocity impact response on hybrid Kevlar composite laminates. In recent years natural fibers are replacing synthetic ones due to their high 
flexibility, low cost and recyclability. Hybrid composites were brought up to increase the low mechanical strength of natural fibers. This hybrid will lead to greater mechanical strength and biodegradability, which is good for the environment. Thickness has an effect on the impact dynamics. Hybrid components are light weight and low cost, hence advantageous. In this authors are reported, about the fabrication of material with different layer laminate in the ratio 3:1:3 (Kevlar: kenaf: Kevlar).

This hybrid composite had a good tensile property. A seven layer kenaf and a seven layer Kevlar was made for comparison. The performance of full kenaf is lesser than the performance of full Kevlar. A low velocity impact test was given for all the above specimens. According to the results, the seven layer laminate only with stood an impact energy of 30 Joules. On the other hand, the hybrid had the mechanical properties and performance of full Kevlar and greater mechanical properties than kenaf composites. By these tests we can conclude that the Kevlar-kenaf hybrid composites can be used as an alternative[11].

Moreover, carbon fibers having excellent contenders for establishing huge strength biomaterials and they have good stress transfer and electrical characteristics it can increase tissue forming. Since carbon fibers possess these properties, tubular carbon layered with poly (methyl-methacrylate) were studied as it can be adopted for internal fixation of bones. In order to make the carbon ductile, ductile Kevlar was enumerated to the composite. Tailorable braiding technology was adopted to make the tubular Kevlar by modifying fiber orientation in the composite. The results from the experiment revealed that with enhancing braiding criterion, mechanical properties near to bone characteristics can be made. Superior capability of stress distribution on composite was achieved since Kevlar braid's physical properties, fiber composite distribution and diameter consistency. Adding PMMA matrix and graphene Nano plates together, it improved composite quality. Hence, it could be adopted as an implant [12]

In this work authors are reported about the execution and examine the characteristics of the novel auxetic Kevlar composite. This research was particularly based on fracture and impact characteristics. To analyses and differentiate, Kevlar interlinked composite was used along with polyurethane analysis and in the absence of it. Short nylon fibers of two distinguished fiber measurement and 3 distinguished fiber distinguished fiber densities were converged. These composites were then fabricated. After the analysis of fracture properties, it was seen that auxetic Kevlar had comprehensive increase of $225 \%$ in comparison with usual interlinked Kevlar for fracture toughness. Moreover, auxetic Kevlar improved by $577 \%$ using converging under initiation toughness. It displayed comprehensive decrease in impaired area in comparison with interlinked correspondent for impact testing. Contrarily, decrease in impaired area determined the decrease in impact energy absorption[13].

\section{RESULTS AND DISCUSSION}

Thereafter, development of Kevlar composite in improving oil and gas ducts because of their exclusive properties. In order to choose the ultimate mechanical performance of laminated Kevlar nanocomposite, curing is a primary objective. There are various criterion during hot pressing that might have a toll on bonding of epoxy matrix and behavioral synergy of the fiber and epoxy. This current research is being carried out to examine most favorable curing conditions. There were three distinct Nano fillers used for this research namely multi walled carbon nanotubes, silicon carbide and aluminum oxide which were integrated in distinct weight ratio. After the analysis, it was seen that the most favorable curing temperature was 170 degree centigrade to increase the mechanical properties. The observation under 100 degree centigrade was that there was the removal of outer layer of the Kevlar. By these tests, we can conclude that by better use of curing and Nano fillers, mechanical strength of composites increases[14].

Moreover, for harvesting body motion energy an elemental component wearable piezoelectric Nano wire generator is used this is called a micro fiber Nano wire hybrid structure. PENG uses Nano wire hybrid structure for different access combining surface coating and plasma etching technique. These techniques are described to intensify the mechanical accuracy of Kevlar micro fiber. As a result the hybrid structure will gain high resistance firmness and durability. The Kevlar fibers coated Zno Nano wire are appropriate to 2D Nano generator based on coupled piezoelectric and semi conducting properties of zinc oxide. These Zno Nano wire covered fibers were used to assemble a high stability 2D Nano generator.2D Nano generator later developed as self-powered system to detect UV intensity[15].

The carbon Kevlar and glass fiber reinforced composite are assembled using fused deposition modeling additive assembling technique. 3D printing system were used to fabricate nylon composite. The mechanical work of composite is to be made in tension flexure. Later the effect of the fabric orientation, fiber type and volume chunk was investigated. This determine that the carbon fiber generates largest in mechanical durability. Its strength was higher than with Nano reinforced nylon polymer. As the result the glass exhibit maximum efficiency in tensile durability. The study says that the nylon composite durability was in the following order: carbon fiber $>$ glass fiber $>$ Kevlar fiber [12]. In this we have discussed about the various process that is used for improve the quality of Kevlar composite. The combinations with other material, treatments and no. of layer used in fabrication also help to increase the Kevlar properties.

\section{CONCLUSIONS}

1. The anisotropic nature of Kevlar fiber provides very high ratio of tensile to compression strength.

2. The hybrid and treated Kevlar offered improved compressive strength, flexural, modulus and thermal properties.

3. Hybridization of Kevlar with new, natural and environment friendly fiber still need to explore. 


\section{A RESEARCH ON KEVLAR AND HYBRID KEVLAR COMPOSITES; A REPORT}

4. Still need to explore the new effective design of technique without affecting the mechanical properties of the composite.

5. Still need to explore the new mechanical and electrical application of the Kevlar composite.

6. Still need to improve the delamination properties of Kevlar during the machining operation.

\section{ACKNOWLEDGEMENT}

The manuscript is prepared by taking assistance from Accendere Knowledge Management Services Pvt. Ltd. We are thankful to them. We also express our gratitude to our teachers and mentor for guiding us throughout the work.

\section{REFERENCES}

1. P. Kumar and A. Kumar, "Geometrical quality evaluation in laser cutting of Inconel-718 sheet by using Taguchi based regression analysis and particle swarm optimization," Infrared Phys. Technol., vol. 89, pp. 369-380, 2018.

2. P. Kumar and A. Kumar, "Parametric optimization of multiple quality characteristics in laser cutting of Inconel-718 by using hybrid approach of multiple regression analysis and genetic algorithm," Infrared Phys. Technol., vol. 91, pp. 220-232, 2018.

3. P. K. Shrivastava and A. K. Pandey, "Multi-Objective Optimization of Cutting Parameters during Laser Cutting of Titanium Alloy Sheet using Hybrid approach of Genetic Algorithm and Multiple Regression Analysis," in Materials Today: Proceedings, 2018, vol. 5, no. 11, pp. 24710-24719.

4. P. K. Shrivastava and A. K. Pandey, "Optimization of Machining Parameter during the Laser Cutting of Inconel-718 Sheet Using Regression Analysis based Particle Swarm Optimization Method," in Materials Today: Proceedings, 2018, vol. 5, no. 11, pp. 24167-24176.

5. T. J. Kang, K. H. Hong, and M. R. Yoo, "Preparation and properties of fumed silica/Kevlar composite fabrics for application of stab resistant material," Fibers Polym., vol. 11, no. 5, pp. 719-724, 2010.

6. S. Saikrasun, T. Amornsakchai, C. Sirisinha, W. Meesiri, and S. Bualek-Limcharoen, "Kevlar reinforcement of polyolefinbased thermoplastic elastomer," Polymer (Guildf)., vol. 40, no. 23, pp. 6437-6442, 1999.

7. M. Mukherjee, C. K. Das, A. P. Kharitonov, K. Banik, G. Mennig, and T. N. Chung, "Properties of syndiotactic polystyrene composites with surface modified short Kevlar fiber," Mater. Sci. Eng. A, vol. 441, no. 1-2, pp. 206-214, 2006.

8. H. Chouhan, N. Asija, S. A. Gebremeskel, and N. Bhatnagar, "Effect of Specimen Thickness on High Strain Rate Properties of Kevlar/Polypropylene Composite," Procedia Eng., vol. 173, pp. 694-701, 2017.

9. Y. Z. Wan, Y. L. Wang, F. He, Y. Huang, and H. J. Jiang, "Mechanical performance of hybrid bismaleimide composites reinforced with three-dimensional braided carbon and Kevlar fabrics," Compos. Part A Appl. Sci. Manuf., vol. 38, no. 2, pp. 495-504, 2007.

10. Q. He, S. Cao, Y. Wang, S. Xuan, P. Wang, and X. Gong, "Impact resistance of shear thickening fluid/Kevlar composite treated with shear-stiffening gel," Compos. Part A Appl. Sci. Manuf., vol. 106, pp. 82-90, 2018.

11. M. F. Ismail, M. T. H. Sultan, A. Hamdan, and A. U. M. Shah, "A study on the low velocity impact response of hybrid Kenaf-Kevlar composite laminates through drop test rig technique," BioResources, vol. 13, no. 2, pp. 3045-3060, 2018.

12. S. Rajesh, B. Vijaya Ramnath, C. Elanchezhian, M. Abhijith, R. Dinesh Riju, and K. Kathir Kishan, "Investigation of
Tensile Behavior of Kevlar Composite," Mater. Today Proc. vol. 5, no. 1, pp. 1156-1161, 2018.

13. F. Nasiri, S. Ajeli, D. Semnani, M. Jahanshahi, and R. Emadi, "Design, fabrication and structural optimization of tubular carbon/Kevlar ${ }^{\circledR} / \mathrm{PMMA} /$ graphene nanoplate composite for bone fixation prosthesis," Biomed. Mater., vol. 13, no. 4, 2018.

14. M. C. Lin, C. W. Lou, J. Y. Lin, T. A. Lin, and J. H. Lin, "Mechanical property evaluations of flexible laminated composites reinforced by high-performance Kevlar filaments: Tensile strength, peel load, and static puncture resistance," Compos. Part B Eng., vol. 166, no. April 2018, pp. 139-147, 2019.

15. L. Zhang et al., "A High-Reliability Kevlar Fiber-ZnO Nanowires Hybrid Nanogenerator and its Application on SelfPowered UV Detection," Adv. Funct. Mater., vol. 25, no. 36 , pp. 5794-5798, 2015. 